Published in final edited form as:

Clin Genitourin Cancer. 2015 October ; 13(5): 453-460. doi:10.1016/j.clgc.2015.05.003.

\title{
Phase I/II Trial of Paclitaxel Plus Ifosfamide Followed by High- Dose Paclitaxel, Ifosfamide, and Carboplatin (TI-TIC) with Autologous Stem Cell Reinfusion for Salvage Treatment of Germ Cell Tumors
}

\author{
Darren R. Feldman ${ }^{1,2}$, llya Glezerman ${ }^{1,2}$, Sujata Patil ${ }^{3}$, Lindsay Van Alstine ${ }^{1}$, Dean F. \\ Bajorin $^{1,2}$, Patricia Fischer ${ }^{1}$, Amanad Hughes ${ }^{1}$, Joel Sheinfeld ${ }^{4}$, Manjit Bains ${ }^{4}$, Lilian \\ Reich $^{1,2}$, Kaitlin Woo ${ }^{3}$, Sergio Giralt ${ }^{1,2}$, George J. BosI ${ }^{1,2}$, and Robert J. Motzer ${ }^{1,2}$ \\ ${ }^{1}$ Department of Medicine, Memorial Sloan Kettering Cancer Center, 1275 York Avenue, New York, \\ NY, 10065, USA \\ 2Department of Medicine, Weill Cornell Medical College, 1300 York Avenue, New York, NY, \\ 10065, USA \\ ${ }^{3}$ Department of Epidemiology and Biostatistics, Memorial Sloan Kettering Cancer Center, 1275 \\ York Avenue, New York, NY, 10065, USA \\ ${ }^{4}$ Department of Surgery, Memorial Sloan Kettering Cancer Center, 1275 York Avenue, New York, \\ NY, 10065, USA
}

\begin{abstract}
Purpose-Salvage high-dose chemotherapy with autologous stem cell transplant (ASCT), consisting of 2-3 sequential cycles of high-dose (HD) carboplatin and etoposide (CE) can achieve durable remissions in approximately one-half of relapsed germ cell tumor patients. To improve upon these results and based on success with paclitaxel, ifosfamide, and cisplatin (TIP) as salvage conventional-dose chemotherapy, we conducted a phase I/II trial of HD paclitaxel plus ifosfamide (TI), substituting carboplatin for cisplatin to allow dose escalation.
\end{abstract}

Patients and Methods-Treatment consisted of 1-2 cycles of TI and granulocyte colony stimulating factor for stem cell mobilization followed by three cycles of HD TI plus carboplatin (TIC) with ASCT every 21-28 days. Twenty-six patients were enrolled. For phase I, a standard $3+3$ dose-escalation design was used.

Results-With no dose-limiting toxicities observed, the maximum tolerated dose (MTD) was not reached and the highest prespecified dose level (paclitaxel $250 \mathrm{mg} / \mathrm{m}^{2}$, ifosfamide $9990 \mathrm{mg} / \mathrm{m}^{2}$,

Corresponding author: Darren R. Feldman, MD, Genitourinary Oncology Service, Division of Solid Tumor Oncology, Department of Medicine, Memorial Sloan Kettering Cancer Center, 1275 York Avenue, New York, NY, 10065, USA, Phone: 646-422-4491, Fax: 212-988-0701, feldmand@mskcc.org.

Conflict of Interest Statement: The authors declare no conflict of interest.

Publisher's Disclaimer: This is a PDF file of an unedited manuscript that has been accepted for publication. As a service to our customers we are providing this early version of the manuscript. The manuscript will undergo copyediting, typesetting, and review of the resulting proof before it is published in its final citable form. Please note that during the production process errors may be discovered which could affect the content, and all legal disclaimers that apply to the journal pertain. 
carboplatin area under the curve $=24$ ) was considered the MTD. Phase II employed a Simon twostage design to estimate the complete response (CR) rate at the MTD. With 7/11 phase II patients achieving CR, efficacy was demonstrated. However, three patients developed delayed chronic kidney disease, resulting in premature trial closure.

Conclusion-TI-TIC was active in relapsed GCT but emergent chronic renal impairment, possibly from overlapping ifosfamide and carboplatin, preclude its further use. TI-CE, consisting of two cycles of TI plus three cycles of HD CE remains the standard-of-care HD chemotherapy regimen at Memorial Sloan Kettering Cancer Center.

\section{Keywords}

high-dose chemotherapy; autologous stem cell support; nephrotoxicity; testicular cancer

\section{Introduction}

Germ cell tumors (GCT) are the most common tumor to affect adolescent and young adult men in developed countries. Despite a high overall cure rate, up to $30 \%$ of patients with advanced GCT fail to achieve durable remissions with first-line chemotherapy. ${ }^{1}$ These patients require salvage approaches, consisting of either four cycles of conventional-dose chemotherapy (CDCT) or 2-3 cycles of sequential high-dose chemotherapy (HD CT) followed by autologous stem cell transplantation (ASCT). After progression to salvage CD CT, HD CT remains a curative treatment option.

Carboplatin and etoposide (CE) comprise the backbone of HD CT for GCT. As salvage therapy, complete responses (CR) can be achieved in 40-70\% of patients with remissions remaining durable in $30-63 \% .{ }^{2-6}$ Improving upon these results is a research priority.

Our previous studies demonstrated synergistic activity of paclitaxel, ifosfamide, and cisplatin (TIP) in both GCT cell lines and patients. ${ }^{7}$ In a phase II trial of TIP as initial salvage therapy for GCT patients with favorable features (gonadal primary site and favorable response to first-line chemotherapy), improved efficacy was observed compared to historical results with regimens such as vinblastine, ifosfamide, and cisplatin. ${ }^{8}$ Even accounting for the favorable population in this study, TIP appeared to have superior activity. Our hypothesis was that high-dose (HD) TIP could improve upon results with prior HD CT regimens. Therefore, we conducted a phase I/II study of HD paclitaxel plus ifosfamide, with substitution of carboplatin for cisplatin to allow dose escalation.

\section{Patients and Methods}

\section{Patients}

Eligible patients included men and women $\geq 18$ years of age with GCT of any primary site (including the central nervous system) that was histologically-confirmed at Memorial Sloan Kettering Cancer Center (MSKCC) and progressive disease (PD) after $\geq 1$ cisplatin-based chemotherapy regimen. Evidence of PD included worsening radiographic findings, increase in elevated serum tumor markers (STMs), or known residual disease after postchemotherapy surgery. Adequate bone marrow (white blood cells $\geq 3,000 /$ ul, platelets 
$\geq 100,000 / \mathrm{ul}$ ) and organ function (12-hour urine creatinine clearance $>50 \mathrm{cc} / \mathrm{min}$, aspartate transaminase/alanine transaminase $<2 \times$ the upper limit of normal [ULN] and bilirubin $<1.5 \times \mathrm{ULN}$ ) was also required. Exclusion criteria included prior HD CT or positive HIV or human T-lymphotropic virus serology. All patients provided informed consent.

\section{Study Design and Treatment}

This prospective, single-institution, phase I/II trial was approved by the MSKCC institutional review board. Pretreatment evaluation for HD CT at our center has previously been described. ${ }^{9}$ For simplicity, the regimen was divided into two parts. In Part A, one or two cycles of paclitaxel $\left(200 \mathrm{mg} / \mathrm{m}^{2}\right.$ over 3 hours on day 1$)$ plus ifosfamide $\left(2000 \mathrm{mg} / \mathrm{m}^{2} \mathrm{IV}\right.$ daily from days 1 to 3 ) mixed 1:1 with mesna were administered every 14-21 days. Subcutaneous injections of $10 \mathrm{mcg} / \mathrm{kg} /$ day of granulocyte colony stimulating factor (G-CSF) commenced 6 hours after completion of ifosfamide on day 3 to facilitate stem cell mobilization. If sufficient hematopoietic stem cells were collected with cycle 1 of Part A, the second cycle was omitted and patients proceeded directly to Part B. In Part B, 3 cycles of paclitaxel, ifosfamide, and carboplatin (TIC) were administered with ASCT every 21-28 days (Part B, cycles 1-3). A minimum of $2 \times 10^{6} \mathrm{CD} 34+$ cells $/ \mathrm{kg}$ were reinfused on day 5 (day 0 ) followed by pegylated G-CSF to facilitate engraftment (Table 1).

Part A was administered at identical doses to all patients. A standard 3+3 dose-escalation design was used for Part B with five dose levels (Table 2). Ifosfamide dosing varied between cohorts 1-3, paclitaxel was increased for dose level 4, and carboplatin was increased for dose level 5. Carboplatin dosing was by target area under the curve (AUC); all other drugs were dosed by body surface area.

Supportive care measures included prophylactic antifungal troches and fluoroquinolones during Part B. Management of complications included platelet and RBC transfusions. Neutropenic fever was treated with broad-spectrum antibiotics.

\section{Evaluation of Response and Toxicity}

Responses were assessed using STMs, imaging, and if applicable, surgical and pathologic findings. Responses were categorized as CR, partial response with negative tumor markers (PR-negative) or incomplete response (IR) as previously described. ${ }^{9}$ STMs were obtained weekly while cross-sectional imaging with CT or magnetic resonance imaging (MRI) was obtained at baseline, before Part B, and at chemotherapy completion. Serum creatinine was monitored throughout treatment with 12-hour urine creatinine clearance obtained at baseline and before Part B.

\section{Statistics}

The phase I primary endpoint was to determine the MTD of HD TIC. Dose-limiting toxicities (DLTs) consisted of any grade IV non-hematologic toxicity or any grade V toxicity occurring within 21 days of initiation of HD cycle 1 (Part B). The MTD was defined as the highest dose level at which $<2 / 6$ patients experienced a DLT. 
The phase II primary endpoint was to determine the efficacy (CR rate with 95\% confidence interval) of the MTD of TI-TIC using a Simon two-stage design ${ }^{10}$ to discriminate between CR rates of $60 \%$ and $80 \%$ with type I and type II errors of $10 \%$. The lower limit of $60 \%$ was chosen based on the target population being a mixture of patients with favorable and unfavorable prognostic features. ${ }^{3}$ Six patients treated at the MTD during phase I were also included in phase II. Initially, 11 patients would be enrolled in the first Simon stage with an additional 27 accrued in the second stage if $\geq 7 / 11$ achieved a CR.

Secondary endpoints included progression-free survival (PFS), defined as time from treatment start until PD (including IR), death, or last follow-up; and overall survival (OS), defined as time from treatment start until death or last follow-up. Adverse events, according to the National Cancer Institute Common Terminology Criteria for Adverse Events (CTCAE) v3.0, were described by frequency and grade by cycle with the maximum grade over all cycles used as the summary measure per patient. PFS and OS were estimated using the Kaplan-Meier method.

\section{Results}

\section{Patient Characteristics}

Characteristics of the 26 patients ( 25 men) enrolled on the study are shown in Table 3. Twenty-one (81\%) had nonseminoma and $5(19 \%)$ had seminoma. Testis $(\mathrm{n}=15)$ and mediastinum $(\mathrm{n}=6)$ were the most common primary sites. Best response to first-line chemotherapy was an IR in 19, CR in five, and PR-negative in two. Six of seven with prior $\mathrm{CR}$ or PR-negative relapsed within six months and the remaining patient had a late relapse (>2 years). Most patients had received one prior line of chemotherapy but three also required one cycle of salvage TIP for cytoreduction of rapidly progressive disease prior to enrollment. However, none had progressed to a full course of salvage CDCT.

\section{Cycles of Treatment Administered}

Twenty-seven cycles of TI plus G-CSF (Part A) were administered to the 26 patients with 24 achieving adequate stem cell collection with one cycle. One patient required two cycles with plerixafor added to cycle 2 to ensure sufficient collection. One patient had such a poor collection with his first TI cycle that a second cycle was deemed futile and he was removed from the study. Two additional patients also did not receive Part B, one for an infectious complication during stem cell mobilization, and one because of PD from teratoma with malignant transformation (Figure 1).

Of 23 patients treated with HD TIC, 17 (74\%) received all three planned HD cycles and six (26\%) received two. Omission of the third cycle was due to toxicity in 5/6 and due to PD in the remaining patient.

\section{Toxicity and Maximal Tolerated Dose (MTD) in Phase I}

In phase I, 21 patients were enrolled into one of five dose cohorts (Table 2). All patients were evaluable for toxicity during Part A (TI). However, three patients who did not receive HD TIC were not evaluable for DLTs and were replaced (one each in cohorts 1, 4, and 5). 
With no DLTs among the 18 evaluable patients, the MTD was not reached and was, therefore, considered cohort 5 (the top dose-level tested). Six patients in phase I were enrolled into the MTD cohort, and were also considered part of phase II.

Part A (TI plus G-CSF) was well-tolerated with few grade 3 or 4 (G3/4) adverse events other than expected hematologic toxicity. There was one neutropenic fever and three patients developed G3 hyperglycemia (all asymptomatic).

Toxicities affecting phase I patients in Part B (HD TIC) are summarized in Table 4. All patients experienced G4 myelosuppression, as expected. In addition, 7/18 developed neutropenic fever and 17/18 experienced G3/4 anemia (11 G3; 6 G4) with no significant difference between dose cohorts. Other G3/4 toxicities of interest included hypokalemia in 13 , hypophosphatemia in 12, elevation of ALT in nine, and hyponatremia in seven. G3 toxicities that increased with dose-escalation included orthostatic hypotension $(\mathrm{n}=3)$, renal failure ( $\mathrm{n}=2$, see below), and liver function test abnormalities. There were no treatmentrelated deaths.

\section{Efficacy}

Eleven patients were treated at the MTD and evaluable for response, including six from phase I. Best response included CR in seven, PR-negative in one, and IR in three. Although the first Simon stage efficacy endpoint was met (allowing enrollment of 27 additional patients), irreversible renal insufficiency in $3 / 23$ patients led to premature study termination.

Four of 11 patients treated at the MTD died from progressive GCT, including the three with IRs and one CR who relapsed after two months. One additional patient relapsed from a PRwith a solitary brain metastasis six months post-HD CT and was successfully treated with surgery and stereotactic radiation. He remains alive and progression-free for more than 15 months.

Among phase II patients, the 1-year PFS was 62.3\% (95\% CI 38.9-100\%) with median followup of 13.7 months and the 1-year OS was 70\% (95\% CI 46.7-100\%) with median followup for survivors of 22 months. In total, 12 of 26 patients accrued to the study died; the remaining 14 were all alive and disease-free at last follow-up. Among all patients (phase I and II), two of three with a retroperitoneal primary site and one of six with a mediastinal primary site achieved long-term disease-free status as did the one female patient. The patient with pineal gland GCT achieved PR-negative status after TI-TIC but relapsed about 8 months later and ultimately died of disease.

\section{Renal Failure}

Seven patients developed $\geq$ grade 2 acute kidney injury (AKI) according to CTCAE v3.0. Of these, three developed chronic kidney disease (CKD), as illustrated in Figure 2. Patient 1 had primary retroperitoneal seminoma with an atrophic left kidney due to chronic hydronephrosis, contributing only $18 \%$ to global renal function. His baseline creatinine was $1.3 \mathrm{mg} / \mathrm{dL}$ (ULN $1.3 \mathrm{mg} / \mathrm{dL}$ ). Patient 2 had testicular nonseminoma with baseline creatinine of $1.6 \mathrm{mg} / \mathrm{dL}$ and patient 3 hadprimary mediastinal non-seminoma with baseline creatinine of $1.1 \mathrm{mg} / \mathrm{dL}$. None of the three developed AKI during Part A. In all cases, AKI first 
occurred approximately one week into cycle 1 of Part B, followed by recovery of creatinine to baseline over the next 2 weeks. AKI ensued again during cycle 2 with a similar timeframe but there was much more limited recovery followed by a slow progressive rise in creatinine. Patient 1 had the most rapid progression of CKD, requiring dialysis by 9 months post completion of high-dose TIC. At last evaluation, approximately 3.5 years following completion of HD TIC, patient 2 had a creatinine of $5.1 \mathrm{mg} / \mathrm{dl}$. He has not required hemodialysis but is being evaluated for renal transplant. Patient 3 relapsed at 4 months following completion of HD TIC and passed away about 9 months later. His creatinine was continuing to rise and had reached $3.0 \mathrm{mg} / \mathrm{dl}$ at the evaluation closest to his death.

In addition to AKI with sub-nephrotic range proteinuria, all three patients developed significant metabolic derangements consistent with proximal renal tubular dysfunction (Fanconi syndrome) including grade 3/4 hypophosphatemia, grade 3/4 hypokalemia, glucosuria with normal serum glucose levels, and metabolic acidosis. All three also had intermittent microscopic hematuria attributed to ifosfamide bladder toxicity and they all experienced symptomatic postural hypotension with mild hyponatremia and renal salt wasting, treated with sodium chloride tablets, midodrine and fludrocortisone. Orthostatic symptoms resolved in patients 1 and 2 at one and four months post-HD CT, respectively, whereas patient 3 remained orthostatic until his last visit. No renal biopsies were performed due to concerns about chemotherapy-induced thrombocytopenia, a solitary functioning kidney in patient 1 , and relative certainty about the causal effect of chemotherapy given the timing of renal failure.

\section{Discussion}

Based on excellent outcomes with TIP as initial salvage CDCT, ${ }^{8}$ this phase I/II study was designed to evaluate a HD TIP regimen with substitution of carboplatin for cisplatin. HD TIC was successfully dose-escalated to the maximum dose cohort (paclitaxel $250 \mathrm{mg} / \mathrm{m}^{2}$, ifosfamide $9990 \mathrm{mg} / \mathrm{m}^{2}$, and carboplatin AUC $=24$ per cycle), which was then tested in phase II. Although efficacy was observed, irreversible renal insufficiency among 3/23 patients led us to terminate the trial prematurely. In particular, one patient in dose level 4 and two at the MTD developed CKD. While two of these three patients had mildly elevated baseline creatinine levels, other patients with similar starting values did not develop nephrotoxicity.

HD CT with ASCT constitutes an important part of salvage therapy for advanced GCT. Regimens consisting of 2-3 cycles of sequential HD carboplatin and etoposide result in durable remissions in 30-63\%..$^{2-6}$ While the optimal initial salvage approach (CDCT or HD CT) remains controversial, HD CT represents the only curative systemic option following failure of salvage CD CT. ${ }^{11}$ In addition, HD CT offers curative potential for patients with primary mediastinal NSGCT or an IR to first-line chemotherapy, who have $<10 \%$ cure rates with salvage CD CT. ${ }^{3,12}$ Nevertheless, since many patients are not cured with current salvage HD CT regimens, development of novel HD CT approaches represents an important area of clinical research. 
Several prior studies incorporated ifosfamide into HD CT programs for GCT, particularly in combination with carboplatin and etoposide (ICE), with only a few observing excess nephrotoxicity. Of $135 \mathrm{GCT}$ patients treated with HD ICE (ifosfamide $5-10 \mathrm{~g} / \mathrm{m}^{2} /$ cycle) by the German Testicular Cancer Study Group (GTCSG), 39 (29\%) experienced either a $\geq 50 \%$ decline in glomerular filtration rate (GFR) using the Cockroft-Gault equation or increase in creatinine by $\geq 1.0 \mathrm{mg} / \mathrm{dL} .{ }^{13}$ Twelve (8\%) patients required hemodialysis (two long-term) and $5 / 12$ died from toxicity. Nephrotoxicity was most common at the highest carboplatin doses $\left(1750 \mathrm{mg} / \mathrm{m}^{2}\right.$ and $\left.2000 \mathrm{mg} / \mathrm{m}^{2}\right)$ but unlike the current study, was reversible in most patients.

In another series of 131 patients with various malignancies, HD ICE (ifosfamide 6-24 g/m $/$ cycle) resulted in peak creatinine levels of $1.6-3.0 \mathrm{mg} / \mathrm{dL}$ in $37 \%$ and $>3.0 \mathrm{mg} / \mathrm{dL}$ in $11 \% .^{14}$ Renal insufficiency was more frequent at ifosfamide doses of $20.1-24 \mathrm{~g} / \mathrm{m}^{2} / \mathrm{cycle}$ vs. $6-17.1$ $\mathrm{g} / \mathrm{m}^{2} / \mathrm{cycle}$, with one patient requiring hemodialysis and two others experiencing delayed CKD.

Fewer studies have evaluated HD TIC. Pal and colleagues studied a regimen of HD paclitaxel, etoposide and carboplatin (TEC) followed by HD TIC (TEC-TIC), observing G3/4 nephrotoxicity in only $1 / 29$ patients who received TIC. ${ }^{15}$ Similar doses of carboplatin (AUC $=21$ over 3 days) and ifosfamide $\left(9000 \mathrm{mg} / \mathrm{m}^{2}\right.$ over 3 days by 30 -minute infusion) were used but with higher paclitaxel doses $\left(350 \mathrm{mg} / \mathrm{m}^{2}\right.$ or $425 \mathrm{mg} / \mathrm{m}^{2} /$ cycle). Excess nephrotoxicity was also not seen with TI-CE, consisting of paclitaxel plus ifosfamide at conventional doses for stem cell mobilization (identical to Part A in the current study) followed by three cycles of HD CE with ASCT. ${ }^{3}$

Given the lack of renal injury with HD CE, we hypothesize that overlapping nephrotoxicity of ifosfamide and carboplatin caused the CKD in our study. This is supported by development of Fanconi syndrome, a classic component of ifosfamide nephrotoxicity ${ }^{16,17}$ in all three CKD patients. However, the reason we observed more frequent CKD than other studies is unclear. Risk factors for ifosfamide nephrotoxicity include higher cumulative doses, prior or concurrent cisplatin treatment, and preexisting renal impairment. ${ }^{17}$ None of these factors were more prevalent in our population. In fact, the HD ICE studies detailed above administered equal or higher doses of ifosfamide and carboplatin, making the degree of CKD we observed unexpected. Moreover, while some have suggested short ifosfamide infusion times predispose to ifosfamide renal injury, evidence supporting this notion is lacking ${ }^{18,19}$ and Pal's use of a 30-minute ifosfamide infusion (but similar or higher TIC doses) without excess nephrotoxicity ${ }^{15}$ further refutes this assertion. The lack of CKD with HD TEC-TIC might instead relate to inclusion of only one HD TIC cycle or omission of ifosfamide from stem cell mobilization, since CKD with TI-TIC occurred uniformly after cycle 2 of TIC and $\geq 3$ cycles of ifosfamide. Furthermore, all 3 patients experiencing CKD following HD cycle 2 had reversible AKI after HD cycle 1.

Our patients also experienced salt-wasting nephropathy, previously described in $10 \%$ of cisplatin-treated patients but rarely with ifosfamide or carboplatin. ${ }^{20,21}$ Thus, ongoing hypotension and renal hypoperfusion could have contributed to CKD severity in our patients. Finally, patient 1 , who required dialysis, had an atrophic kidney, possibly predisposing him 
to ifosfamide nephrotoxicity, given the physiologic similarities (glomerular hyperfiltration and increased toxin exposure) to prior nephrectomy, a known risk factor for ifosfamide nephrotoxicity. ${ }^{22,23}$

In summary, while TI-TIC demonstrated activity in patients with relapsed or refractory GCT, CKD observed at the highest ifosfamide dose led to premature study closure. Thus, TI-CE remains the standard-of-care for salvage HD CT at MSKCC. Although improving upon carboplatin and etoposide HD CT programs is a priority, we do not plan to further investigate the addition of ifosfamide to the HD portion of such regimens. Rather, our focus will be on identifying novel active agents unlikely to yield significant renal (or other nonhematological) toxicity when combined with etoposide and carboplatin and using genetic profiling to understand the molecular underpinnings of response and resistance. Finally, we are currently involved in actively developing a prospective randomized phase III trial comparing the efficacy of HD CT and CD CT as initial salvage treatment.

\section{Acknowledgments}

Supported by the Craig D. Tifford Foundation and the Sidney Kimmel Center for Prostate and Urologic Cancers (New York, NY). We thank Michael Newman and Joyce Tsoi for their editorial review of the manuscript.

Supported by Sidney Kimmel Center for Prostate and Urologic Cancers (NY, NY) and the Craig D. Tifford Foundation

\section{References}

1. Feldman DR, Bosl GJ, Sheinfeld J, et al. Medical treatment of advanced testicular cancer. JAMA. 2008; 299:672-84. [PubMed: 18270356]

2. Einhorn LH, Williams SD, Chamness A, et al. High-dose chemotherapy and stem-cell rescue for metastatic germ-cell tumors. N Engl J Med. 2007; 357:340-8. [PubMed: 17652649]

3. Feldman DR, Sheinfeld J, Bajorin DF, et al. TI-CE high-dose chemotherapy for patients with previously treated germ cell tumors: results and prognostic factor analysis. J Clin Oncol. 2010; 28:1706-13. [PubMed: 20194867]

4. Lorch A, Kleinhans A, Kramar A, et al. Sequential versus single high-dose chemotherapy in patients with relapsed or refractory germ cell tumors: long-term results of a prospective randomized trial. J Clin Oncol. 2012; 30:800-5. [PubMed: 22291076]

5. Lorch A, Kollmannsberger C, Hartmann JT, et al. Single versus sequential high-dose chemotherapy in patients with relapsed or refractory germ cell tumors: a prospective randomized multicenter trial of the German Testicular Cancer Study Group. J Clin Oncol. 2007; 25:2778-84. [PubMed: 17602082]

6. Nichols CR, Andersen J, Lazarus HM, et al. High-dose carboplatin and etoposide with autologous bone marrow transplantation in refractory germ cell cancer: an Eastern Cooperative Oncology Group protocol. J Clin Oncol. 1992; 10:558-63. [PubMed: 1312584]

7. Motzer RJ, Sheinfeld J, Mazumdar M, et al. Paclitaxel, Ifosfamide, and Cisplatin Second-Line Therapy for Patients With Relapsed Testicular Germ Cell Cancer. Journal of Clinical Oncology. 2000; 18:2413-8. [PubMed: 10856101]

8. Kondagunta GV, Bacik J, Donadio A, et al. Combination of paclitaxel, ifosfamide, and cisplatin is an effective second-line therapy for patients with relapsed testicular germ cell tumors. J Clin Oncol. 2005; 23:6549-55. [PubMed: 16170162]

9. Motzer RJ, Mazumdar M, Sheinfeld J, et al. Sequential dose-intensive paclitaxel, ifosfamide, carboplatin, and etoposide salvage therapy for germ cell tumor patients. J Clin Oncol. 2000; 18:1173-80. [PubMed: 10715285] 
10. Simon R. Optimal two-stage designs for phase II clinical trials. Control Clin Trials. 1989; 10:1-10. [PubMed: 2702835]

11. Voss MH, Feldman DR, Motzer RJ. High-dose chemotherapy and stem cell transplantation for advanced testicular cancer. Expert Rev Anticancer Ther. 2011; 11:1091-103. [PubMed: 21806332]

12. Motzer RJ, Geller NL, Tan CC, et al. Salvage chemotherapy for patients with germ cell tumors. The Memorial Sloan-Kettering Cancer Center experience (1979-1989). Cancer. 1991; 67:1305-10. [PubMed: 1703917]

13. Beyer J, Rick O, Weinknecht S, et al. Nephrotoxicity after high-dose carboplatin, etoposide and ifosfamide in germ-cell tumors: incidence and implications for hematologic recovery and clinical outcome. Bone Marrow Transplant. 1997; 20:813-9. [PubMed: 9404920]

14. Agaliotis DP, Ballester OF, Mattox T, et al. Nephrotoxicity of high-dose ifosfamide/carboplatin/ etoposide in adults undergoing autologous stem cell transplantation. Am J Med Sci. 1997; 314:292-8. [PubMed: 9365330]

15. Pal SK, Yamzon J, Sun V, et al. Paclitaxel-based high-dose chemotherapy with autologous stem cell rescue for relapsed germ cell tumor: clinical outcome and quality of life in long-term survivors. Clin Genitourin Cancer. 2013; 11:121-7. [PubMed: 23062817]

16. Rossi R, Pleyer J, Schafers P, et al. Development of ifosfamide-induced nephrotoxicity: prospective follow-up in 75 patients. Med Pediatr Oncol. 1999; 32:177-82. [PubMed: 10064184]

17. Skinner R. Nephrotoxicity--what do we know and what don't we know? J Pediatr Hematol Oncol. 2011; 33:128-34. [PubMed: 21285904]

18. Rossi R, Schafers P, Pleyer J, et al. The influence of short versus continuous ifosfamide infusion on the development of renal tubular impairment. Int J Pediatr Hematol Oncol. 1997; 4:393-9.

19. Boddy AV, English M, Pearson AD, et al. Ifosfamide nephrotoxicity: limited influence of metabolism and mode of administration during repeated therapy in paediatrics. Eur J Cancer. 1996; 32A:1179-84. [PubMed: 8758250]

20. Tscherning C, Rubie H, Chancholle A, et al. Recurrent renal salt wasting in a child treated with carboplatin and etoposide. Cancer. 1994; 73:1761-3. [PubMed: 8156505]

21. Santana VM, Schell MJ, Williams R, et al. Escalating sequential high-dose carboplatin and etoposide with autologous marrow support in children with relapsed solid tumors. Bone Marrow Transplant. 1992; 10:457-62. [PubMed: 1464010]

22. Rossi R. Increased risk of ifosfamide-induced renal Fanconi's syndrome after unilateral nephrectomy. The Lancet (British edition). 1993; 341:755.

23. Rossi R, Godde A, Kleinebrand A, et al. Unilateral nephrectomy and cisplatin as risk factors of ifosfamide-induced nephrotoxicity: analysis of 120 patients. J Clin Oncol. 1994; 12:159-65. [PubMed: 8270973] 


\section{Clinical Practice Points}

- $\quad$ Based on the effectivess of salvage CD TIP in relapsed GCT patients with favorable features, we developed TI-TIC as a HD TIP regimen with substitution of carboplatin for cisplatin to allow dose escalation.

- $\quad$ In the phase I portion of this phase I/II study, HD TIC was successfully dose-escalated to the maximum dose cohort (paclitaxel $250 \mathrm{mg} / \mathrm{m}^{2}$, ifosfamide $9990 \mathrm{mg} / \mathrm{m}^{2}$, and carboplatin AUC = 24 per cycle).

- $\quad$ Although preliminary efficacy was observed at the MTD in phase II with $7 / 11$ patients achieving $\mathrm{CR}$, progressive irreversible renal insufficiency in $3 / 23$ patients led to premature trial termination.

- Given the nephrotoxicity observed in this study, we have no plans to futher investigate salvage regimens combining HD ifosfamide and HD carboplatin.

- $\quad$ TI-CE remains the standard of care HD regimen at MSKCC. 




Figure 1. CONSORT Diagram of Treatment Received 
A
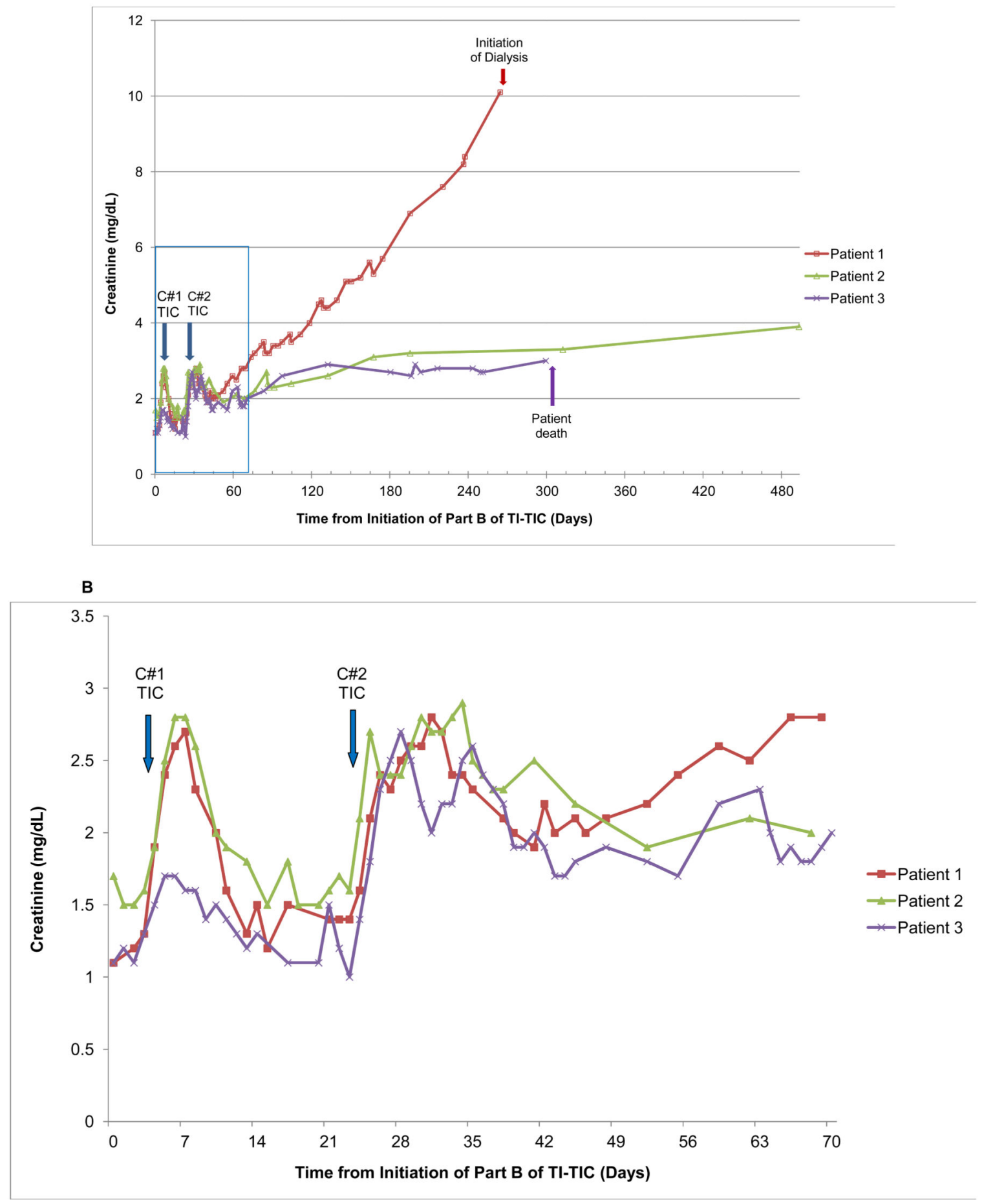

Figure 2.

Graphical illustration of creatinine Levels in the Three Patients who Developed Chronic Renal Insufficiency with High-Dose Paclitaxel, Ifosfamide, and Carboplatin A) From the start of treatment and including long-term followup and B) During treatment only. The insert in Figure 2A is the short-term creatinine changes depicted on a larger scale in Figure $2 \mathrm{~B}$. 
Blue arrows denote the timing of initiation of the two cycles of chemotherapy (C\#1, cycle

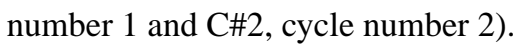






Clin Genitourin Cancer. Author manuscript; available in PMC 2016 October 01. 

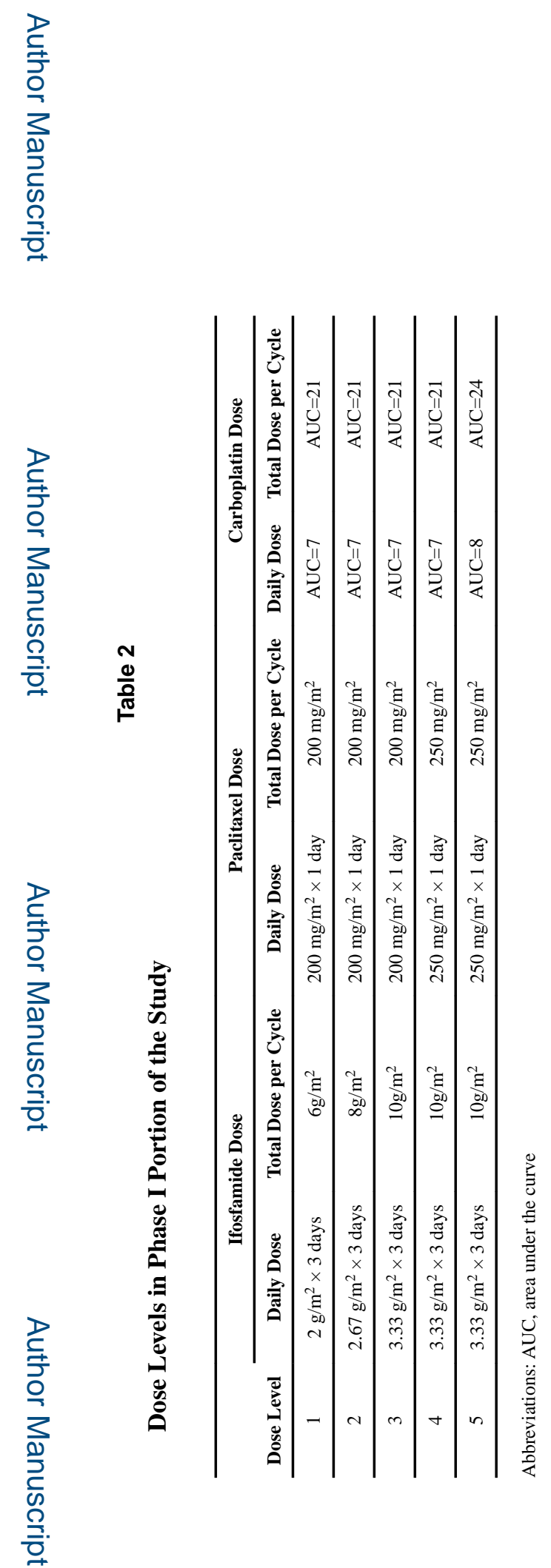

Clin Genitourin Cancer. Author manuscript; available in PMC 2016 October 01. 


\section{Table 3}

Patient Characteristics $(\mathrm{n}=\mathbf{2 6})$

\begin{tabular}{|c|c|c|}
\hline & $\mathbf{N}$ & $\%$ \\
\hline \multicolumn{3}{|l|}{ Sex } \\
\hline Male & 25 & $96 \%$ \\
\hline Female & 1 & $4 \%$ \\
\hline \multicolumn{3}{|l|}{ Age } \\
\hline Median (range) & 31 & $(18.8-47.8)$ \\
\hline \multicolumn{3}{|l|}{ Histology } \\
\hline Non-seminoma & 21 & $81 \%$ \\
\hline Seminoma & 5 & $19 \%$ \\
\hline \multicolumn{3}{|l|}{ Primary Site } \\
\hline Testis & 15 & $58 \%$ \\
\hline Mediastinum & 6 & $23 \%$ \\
\hline Retroperitoneum & 3 & $12 \%$ \\
\hline Ovary & 1 & $4 \%$ \\
\hline Pineal Gland & 1 & $4 \%$ \\
\hline \multicolumn{3}{|l|}{ Sites of Metastatic Disease } \\
\hline Lung & 15 & $58 \%$ \\
\hline Retroperitoneum & 12 & $46 \%$ \\
\hline Mediastinum & 8 & $31 \%$ \\
\hline Liver & 6 & $23 \%$ \\
\hline Other*a & 5 & $19 \%$ \\
\hline \multicolumn{3}{|l|}{ Number of Metastatic Sites } \\
\hline 1 & 3 & $12 \%$ \\
\hline 2 & 7 & $27 \%$ \\
\hline $3+$ & 16 & $61 \%$ \\
\hline \multicolumn{3}{|l|}{ Elevated markers } \\
\hline $\mathrm{HCG}>2.2 \mathrm{mIU} / \mathrm{mL}$ & 16 & $62 \%$ \\
\hline Median elevated (range) & 869.6 & $(4.9-26,652.3)$ \\
\hline $\mathrm{AFP}>15 \mathrm{ng} / \mathrm{mL}$ & 10 & $38 \%$ \\
\hline Median elevated (range) & 160.8 & $(26.4-2,049.5)$ \\
\hline LDH >ULN $(200 / 246)^{b}$ & 8 & $31 \%$ \\
\hline Median × ULN (range) & 1.72 & $1.07-3.44$ \\
\hline Late Relapse & 1 & $4 \%$ \\
\hline
\end{tabular}

Abbreviations: $\mathrm{HCG}=$ human chorionic gonadotropin; AFP = alpha-fetoprotein; $\mathrm{LDH}=$ lactate dehydrogenase; ULN = upper limit of normal

a Other sites of metastases include brain (2) and adrenal gland, kidney, intestine, and bone in 1 each.

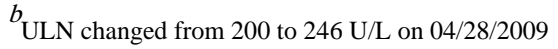




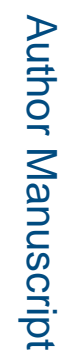

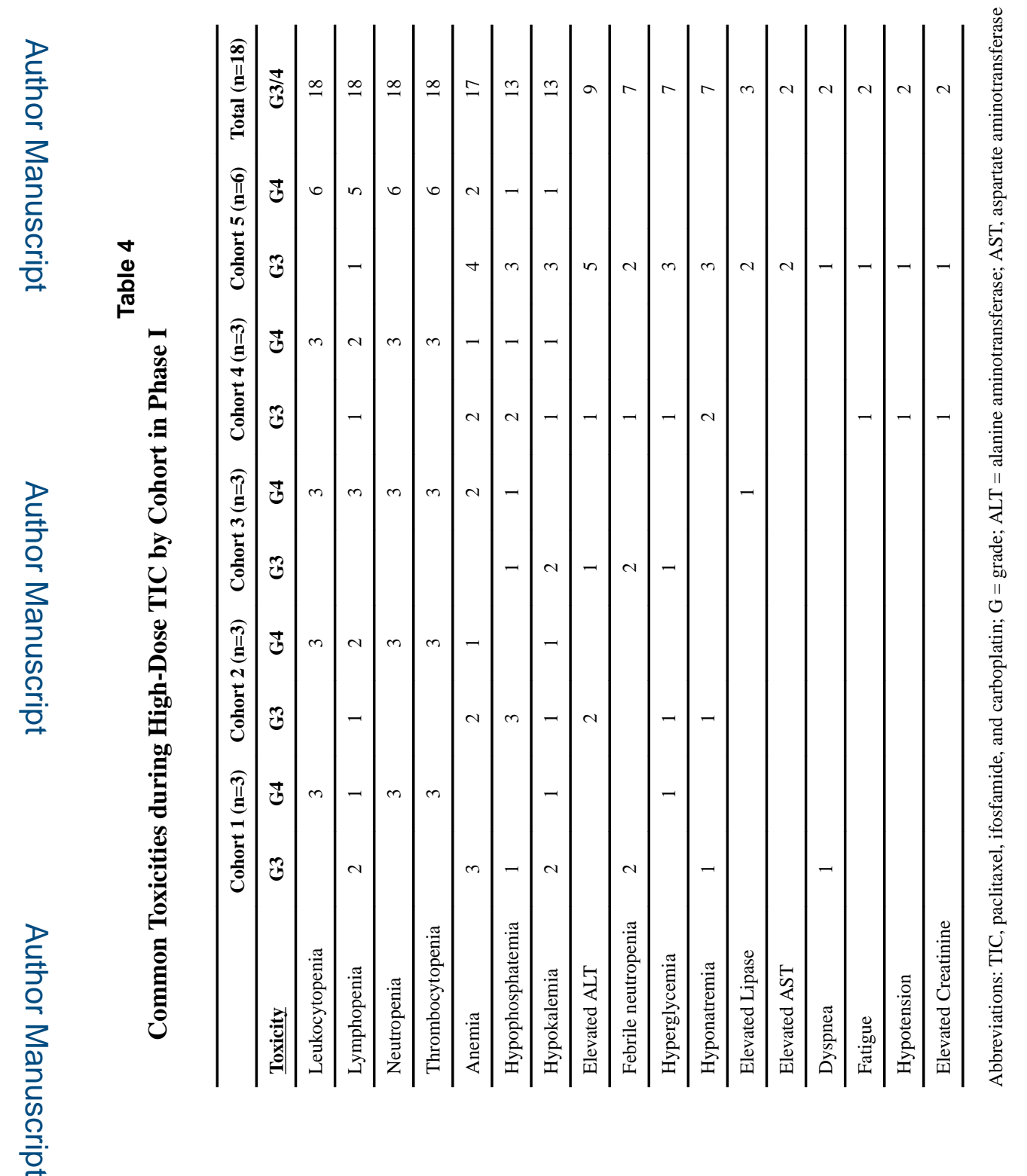

Clin Genitourin Cancer. Author manuscript; available in PMC 2016 October 01. 